Trauma Berufskrankh 2012 · 14[Suppl 2]:208-212 DOI 10.1007/s10039-011-1778-0

Online publiziert: 3. September 2011

c) Springer-Verlag 2011

\section{S.O. Dietz $\cdot$ T.E. Nowak $\cdot$ P.M. Rommens}

Zentrum für Muskuloskelettale Chirurgie, Klinik und Poliklinik für Unfallchirurgie, Universitätsmedizin, Johannes Gutenberg-Universität Mainz

\title{
Wertigkeit des Ellenbogengelenkersatzes
}

Die Behandlung distaler Humerusfrakturen stellt den Unfallchirurgen vor eine große Herausforderung. Bei jungen, gesunden Patienten ist es möglich, das Ellenbogengelenk $\mathrm{zu}$ rekonstruieren und eine für den Patienten zufrieden stellende Funktion zu erreichen. Bei älteren Patienten, die häufig eine Osteoporose und mehrere ernsthafte Begleiterkrankungen aufweisen können, ist die osteosynthetische Versorgung häufiger von Problemen begleitet. In dieser Patientengruppe gewann der alloplastische Ellenbogenersatz im letzten Jahrzehnt zunehmend an Bedeutung.

In dieser Arbeit soll die Wertigkeit des Ellenbogenersatzes in der Traumasituation beschrieben werden.

\section{Epidemiologie}

Aufgrund der demografischen Entwicklung der Bevölkerung rücken Frakturen des Ellenbogengelenks zunehmend in den Fokus des unfallchirurgischen Interesses. Bruchverletzungen des distalen Humerus machen 2-6\% aller Frakturen aus und zeigen insbesondere in der Gruppe der über 60-Jährigen einen deutlichen Anstieg der Inzidenz. Aufgrund der osteoporotischen Knochenstruktur haben Frauen in dieser Altersgruppe ein gegenüber Männern 4-fach erhöhtes Risiko, eine distale $\mathrm{Hu}$ merusfraktur zu erleiden [9, 22]. Luxationsfrakturen des Ellenbogengelenks mit komplexen Frakturen des Processus coronoideus und des Radiuskopfs sind, insbesondere in der am häufigsten betroffenen Gruppe der 50- bis 60-Jährigen, häufig nicht rekonstruktiv zu behandeln [10].

Eine intraartikuläre Trümmerfraktur des distalen Humerus oder eine Luxationsfraktur des Ellenbogens führen im weiteren klinischen Verlauf nicht selten zu einer Einschränkung der Bewegungsumfänge, zu einer Instabilität des Ellenbo- gens und/oder funktionellen Defiziten der gesamten oberen Extremität.

Aufgrund des dünnen Weichteilmantels kann die Wundheilung problematisch sein.

\section{Offene Reposition und interne Stabilisierung}

Bei einer Literaturanalyse der klinischen Ergebnisse von mittels Doppelplattenosteosynthese versorgten distalen Humerusfrakturen (- Abb. 1) zeigten sich bei $74 \%$ (138/187 Patienten) sehr gute und gute Ergebnisse im MEPS („Mayo Elbow Performance Score"). Die Komplikationsrate betrug allerdings über 27\%. Exklusive elektiver Metallentfernungen musste bei $11 \mathrm{~Pa}$ tienten eine Revisionsoperation durchgeführt werden (• Tab. 1). In allen Studien wurde ersichtlich, dass eine höhergradige Verletzung gemäß AO-Klassifikation (AO: Arbeitsgemeinschaft für Osteo-
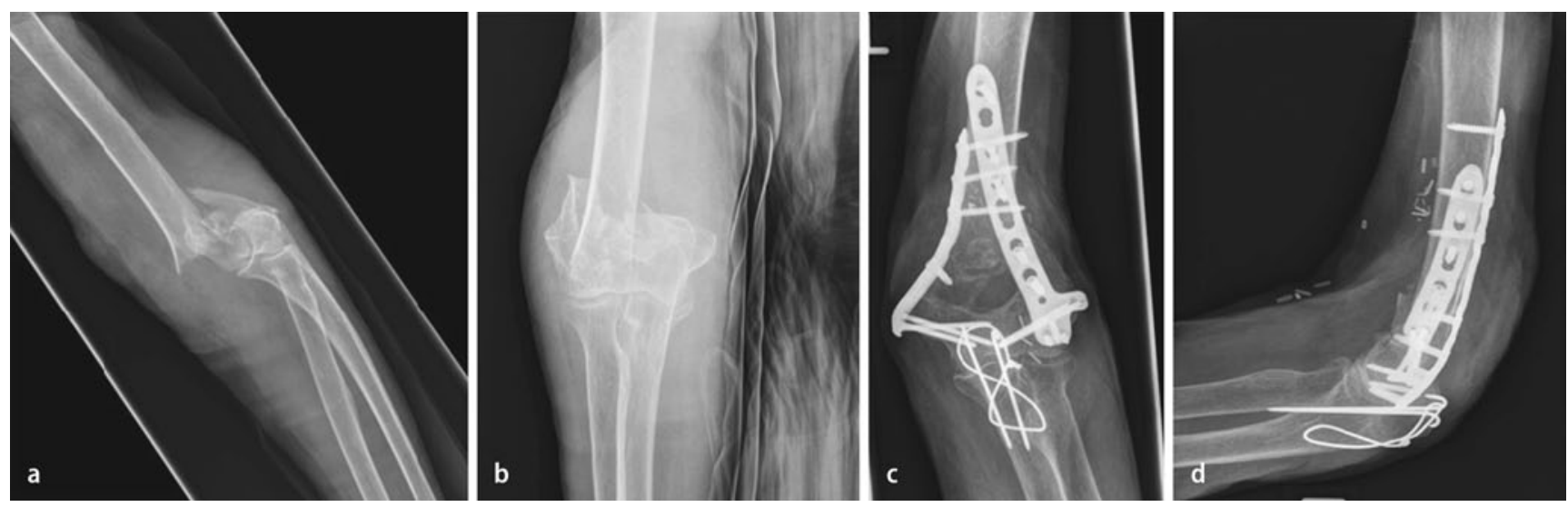

Abb. 1 \ 85-jährige Patientin, Zustand nach Sturz aus dem Stand auf eisglatter Straße, a,b Röntgen präoperativ mit A013C2Fraktur des distalen Humerus, c,d operative Therapie mit offener Reposition und Doppelplattenosteosynthese mit winkelstabilen, anatomisch vorgeformten Platten [LCP DHP (LCP „locking compression plate", DHP: , distal humerus plate“), Synthes, Umkirch, Deutschland], 12 Monate postoperativ subjektive Beschwerdefreiheit, Bewegungsausmaß für Flexion/Extension von $145^{\circ}$ 


\begin{tabular}{|c|c|c|c|c|c|}
\hline Zitat & $\begin{array}{l}\text { Anzahl Patienten } \\
\text { (Durchschnittsalter) }\end{array}$ & $\begin{array}{l}\text { Anzahl sehr guter und } \\
\text { guter Ergebnisse (MEPS) }\end{array}$ & $\begin{array}{l}\text { Bewegungsumfang } \\
\left.\text { [Flexion-Extension }\left({ }^{\circ}\right)\right]\end{array}$ & $\begin{array}{l}\text { MEPS } \\
\text { (Punkte) }\end{array}$ & Komplikationen \\
\hline Korner et al. [14] & $\begin{array}{l}45 \\
\text { (73 Jahre) }\end{array}$ & 26 & 100 & 83 & $\begin{array}{l}\text { 13/45 Patienten } \\
\text { (7 Revisionseingriffe) }\end{array}$ \\
\hline Huang et al. [11] & $\begin{array}{l}14 \\
\text { (78,1 Jahre) }\end{array}$ & 9 & 92 & 83 & $\begin{array}{l}\text { 5/14 Patienten } \\
\text { (1 Revisionseingriff) }\end{array}$ \\
\hline Kaiser et al. [12] & $\begin{array}{l}22 \\
\text { (69 Jahre) }\end{array}$ & 14 & 113 & 84,7 & $4 / 22$ \\
\hline Liu et al. [17] & $\begin{array}{l}32 \\
\text { (68,7 Jahre) }\end{array}$ & 32 & 103 & 93,6 & $11 / 32$ \\
\hline McKee et al. [19] & $\begin{array}{l}15^{\mathrm{a}} \\
\text { (77 Jahre) }\end{array}$ & 8 & 95 & 73 & $8^{a / 15}$ \\
\hline Frankle et al. [6] & $\begin{array}{l}12 \\
\text { (74 Jahre) }\end{array}$ & 8 & 100 & 81 & $\begin{array}{l}\text { 4/12 } \\
\text { (3 sekundäre Prothesen) }\end{array}$ \\
\hline Celli et al. [3] & $\begin{array}{l}18 \\
\text { (55,6 Jahre) }\end{array}$ & 16 & 105,6 & 89,7 & k.A. \\
\hline Greiner et al. [8] & $\begin{array}{l}12 \\
\text { (55,2 Jahre) }\end{array}$ & 11 & 104 & 91 & 3 \\
\hline $\begin{array}{l}\text { Luegmair et al. } \\
\text { [18] }\end{array}$ & $\begin{array}{l}17 \\
\text { (41,5 Jahre) }\end{array}$ & 14 & 112 & 83 & 4 \\
\hline Total & $\begin{array}{l}\text { 187 Patienten } \\
\text { (65,8 Jahre) }\end{array}$ & $\begin{array}{l}\text { 138/187 Patienten } \\
\text { (74\%) }\end{array}$ & $102,7^{\circ}$ & 84,7 Punkte & $\begin{array}{l}\text { 52/187 Patienten } \\
(27,8 \%)\end{array}$ \\
\hline
\end{tabular}

k.A. keine Angabe, MEPS "Mayo Elbow Performance Score"

alnitial wurden 20 Patienten für eine osteosynthetische Versorgung randomisiert, 5 wurden intraoperativ in die Endoprothesengruppe konvertiert

\section{Tab. 2 Klinische Ergebnisse nach endoprothetischer Versorgung distaler Humerusfrakturen}

\begin{tabular}{|c|c|c|c|c|c|}
\hline Zitat & $\begin{array}{l}\text { Anzahl Patienten } \\
\text { (Durchschnittsalter) }\end{array}$ & $\begin{array}{l}\text { Anzahl sehr guter und } \\
\text { guter Ergebnisse (MEPS) }\end{array}$ & $\begin{array}{l}\text { Bewegungsumfang } \\
\left.\text { [Flexion-Extension }\left({ }^{\circ}\right)\right]\end{array}$ & $\begin{array}{l}\text { MEPS } \\
\text { (Punkte) }\end{array}$ & Komplikationen \\
\hline Weber et al. [24] & $\begin{array}{l}12 \\
\text { (66-89 Jahre) }\end{array}$ & 12 & 84,5 & 90,5 & k.A. \\
\hline Kraus et al. [15] & $\begin{array}{l}12 \\
\text { (81 Jahre) }\end{array}$ & 10 & 88 & 81 & $\begin{array}{l}\text { 4 (1-mal aseptische Lockerung, } \\
\text { 3-mal peripheres sensibles Defizit) }\end{array}$ \\
\hline Lee et al. [16] & $\begin{array}{l}7 \\
\text { (72,9 Jahre) }\end{array}$ & 7 & 88,6 & 94,3 & 1 (Spannungsblasen) \\
\hline $\begin{array}{l}\text { Kamineni } \\
\text { u. Morrey [13] }\end{array}$ & $\begin{array}{l}49 \\
\text { (67 Jahre) }\end{array}$ & 46 & 107 & 93 & $\begin{array}{l}17 \\
\text { (9 Revisionseingriffe) }\end{array}$ \\
\hline Chalidis et al. [4] & $\begin{array}{l}11 \\
\text { (79,6 Jahre) }\end{array}$ & 11 & 107 & 90 & 1 \\
\hline Burkhart et al. [2] & $\begin{array}{l}11 \\
\text { (67 Jahre) }\end{array}$ & 10 & 104 & 89,2 & $\begin{array}{l}3 \\
\text { (4 Revisionseingriffe) }\end{array}$ \\
\hline $\begin{array}{l}\text { Cobb } \\
\text { u. Morrey [5] }\end{array}$ & $\begin{array}{l}20 \\
\text { (72 Jahre) }\end{array}$ & 20 & 105 & 95 & $\begin{array}{l}5 \\
\text { (Revisionseingriffe: k.A.) }\end{array}$ \\
\hline Ali et al. [1] & $\begin{array}{l}20 \\
\text { (72 Jahre) }\end{array}$ & 20 & 98 & 92 & 3 \\
\hline McKee et al. [19] & $\begin{array}{l}25^{\mathrm{a}} \\
\text { (78 Jahre) }\end{array}$ & 21 & 107 & 86 & $\begin{array}{l}18 \\
\text { (3 Revisionseingriffe) }\end{array}$ \\
\hline Frankle et al. [6] & $\begin{array}{l}12 \\
\text { (72 Jahre) }\end{array}$ & 12 & 113 & 95 & $\begin{array}{l}5 \\
\text { (3 Revisionseingriffe) }\end{array}$ \\
\hline Total & $\begin{array}{l}179 \text { Patienten } \\
\text { (73,5 Jahre) }\end{array}$ & $\begin{array}{l}\text { 169/179 Patienten } \\
(94,4 \%)\end{array}$ & 100,2 & 90,6 & $\begin{array}{l}\text { 57/179 Patienten (32\%) } \\
19 \text { Revisionseingriffe }\end{array}$ \\
\hline
\end{tabular}

k.A. keine Angabe, MEPS "Mayo Elbow Performance Score"

alnitial waren 20 Patienten für die Versorgung mit einer Totalprothese des Ellenbogengelenks randomisiert. Intraoperativ wurden 5 Patienten aus der mit Osteosynthese in die mit Totalendoprothese versorgte Gruppe von Patienten konvertiert. 
synthesefragen, [20]) mit einem schlechteren Outcome korreliert. Korner et al. [14] stellten zusätzlich fest, dass eine $\mathrm{Ru}$ higstellung der operierten Extremität über 14 Tage zu einer signifikanten Reduktion des postoperativen Bewegungsumfangs führt. Srinivasan et al. [23] berichteten, dass sich die Komplikationsrate von Patienten über 65 Jahren nicht von der jüngerer Patienten unterschied. Ein Vorteil der offenen Reposition und inneren Stabilisierung ist, dass nach knöcherner Konsolidierung der Arm ohne Limitierung beübt werden darf. Bei Durchsicht der Literaturdaten (- Tab. 1) fällt auf, dass sowohl bei Patienten über 65 Jahre $[6,11,12$, $14,17,19]$ als auch bei jüngeren Patienten $[3,8,18]$ überwiegend sehr gute und gute klinische Ergebnisse erreicht werden, wenn die Fraktur für eine Osteosynthese geeignet ist, problemlos knöchern konsolidiert und die Patienten adäquat nachbehandelt werden.

\section{Ellenbogenprothese}

\section{Indikationen und Kontraindikationen}

Es gibt eine Reihe von Faktoren, die eine Osteosynthese erschweren bzw. unmöglich machen. $\mathrm{Zu}$ ihnen gehören:

- multifragmentäre Frakturen mit sehr kleinen Fragmenten, die eine sichere

Fixierung des Osteosynthesematerials unmöglich machen ( $\mathbf{\bullet}$ Abb. 2),

- Verlust der Trochlea durch Zertrümmerung oder Nekrose,

- Verlust des Capitulum humeri durch Zertrümmerung oder Nekrose,

- ausgeprägte Osteoporose und

- sehr distale Frakturen.

Liegt eine solche Frakturmorphologie vor, sollten eine primäre oder sekundäre Frakturversorgung mit einer Endoprothese erwogen werden. Als Indikationen dafür gelten [1]:

- Patienten älter als 75 Jahre mit einer nicht rekonstruierbaren distalen $\mathrm{Hu}$ merusfraktur,

- Patienten mit rheumatoider Arthritis (unabhängig vom Alter),

- Patienten mit deutlich reduzierter Lebenserwartung (unabhängig vom Alter),

Trauma Berufskrankh 2012 • 14[Suppl 2]:208-212 DOI 10.1007/s10039-011-1778-0

(c) Springer-Verlag 2011

\section{S.O. Dietz ·T.E. Nowak · P.M. Rommens Wertigkeit des Ellenbogengelenkersatzes}

\section{Zusammenfassung}

Für die distale, intraartikuläre Humerusfraktur gibt es, im Gegensatz zu den Frakturen des proximalen Femurs, keine eindeutigen Empfehlungen, ab wann ein endoprothetischer Ersatz des Ellenbogengelenks indiziert ist. Hinzu kommt, dass die klinischen Ergebnisse von Ellenbogenprothesen schlechter als diejenigen von Hüftprothesen sind und eine deutliche Gebrauchseinschränkung der Extremität resultiert. Frakturen des distalen $\mathrm{Hu}$ merus betreffen am häufigsten Kinder oder Erwachsene über 60 Jahre. Bei den älteren Patienten kommt erschwerend zur Frakturmorphologie häufig eine osteoporotische Knochenstruktur hinzu. Durch die Einführung moderner, anatomisch vorgeformter, winkelstabiler Implantate konnte die Indikation für die Osteosynthese ausgedehnt werden. Trotz dieser Fortschritte ist eine gelenkerhaltende Chirurgie nicht immer möglich, und die klinischen Ergebnisse bleiben schwierig vorauszusagen. Die vorliegende Arbeit beschäftigt sich mit der Wertigkeit der Endoprothese am distalen Humerus. Hierzu wurden die aktuelle Literatur analysiert und eigene Ergebnisse ausgewertet.

\section{Schlüsselwörter}

Humerus · Humerusfraktur · Frakturosteosynthese $\cdot$ Ellenbogenprothese $\cdot$ Osteoporose

\section{The value of prosthetic replacement of the elbow joint}

\section{Abstract}

Fractures of the distal humerus remain challenging for the treating surgeon. In contrast to proximal femoral fractures, there are no distinct guidelines as to when an endoprosthesis should be used. In addition, the clinical results of elbow prostheses are inferior to those of hip prostheses and the function of the elbow joint is limited after replacement. Children and adults over 60 years of age are predominantly affected by elbow fractures. Bone structure in older patients is often complicated by osteoporosis. The introduction of modern anatomical angle-stable implants has lead to a broadening of the indication for osteosynthesis. Despite these advances, joint-saving surgery is not always possible and the clinical outcome remains incalculable. We review the literature and outline the current recommendations for the diagnosis and surgical therapy of distal humeral fractures in the elderly.

\section{Keywords}

Humerus · Humeral fractures - Osteosynthesis, fracture - Elbow prosthesis - Osteoporosis
- Patienten mit pathologischer Fraktur (unabhängig vom Alter),

- Patienten mit einer degenerativen Ellenbogenarthrose über 60 Jahre,

- Patienten mit posttraumatischer Ellenbogengelenkarthrose über 60 Jahre und

- Pseudarthrose nach distaler Humerusfraktur bei Patienten über 70 Jahre.

Bei der Auswahl der Patienten ist zu berücksichtigen, dass diese sehr kooperativ sein müssen, da sie nach Implantation einer Ellenbogenprothese nicht mehr als $5 \mathrm{~kg}$ tragen und keine für das Ellenbogengelenk sehr belastenden Schwungsportarten wie Tennis oder Golf praktizieren dürfen. Patienten mit hohen Ansprüchen müssen über diese postoperativen Einschränkungen detailliert aufgeklärt werden. Bewährt hat sich die primäre endoprothetische Frakturversorgung bei Patienten mit prätraumatisch bestehender rheumatoider Arthritis oder schmerzhafter degenerativer Arthropathie [5, 6, 21].

Bei den modernen Ellenbogenprothesen handelt es sich um zementierbare Implantate, die bei Bedarf (Instabilität) gekoppelt werden können, ohne die Komponenten auszutauschen, und die eine Varus-Valgus-Bewegung von $7^{\circ}$ erlauben. Dadurch wird die Belastung auf die knöcherne Verankerung vermindert [2]. 

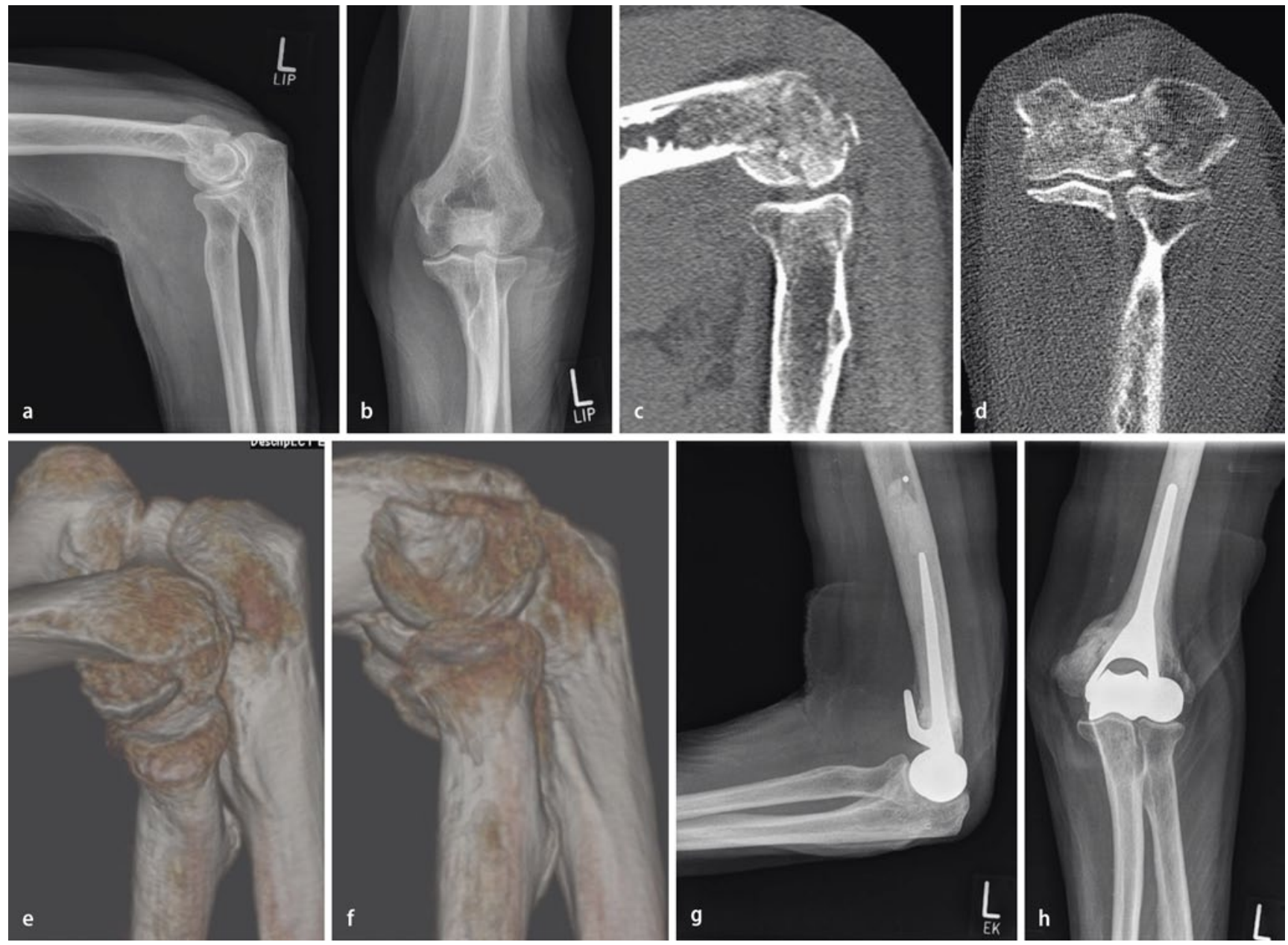

Abb. $2 \Delta$ A013B3.3-Fraktur, a,b konventionelle Bildgebung, Verletzungsschwere nicht ausreichend wiedergegeben, c-f sehr distal gelegene und kleine Fragmente in Computertomographie (c,d) und deren 3D-Rekonstruktion $(\mathbf{e}, \mathbf{f}), \mathbf{g}, \mathbf{h}$ Implantation einer Hemiendoprothese

\section{Ergebnisse}

In der Literatur finden sich zu über $90 \%$ sehr gute und gute klinische Ergebnisse nach primärer Versorgung komplexer Ellenbogenfrakturen mit einer Prothese [1, $2,4,5,6,13,15,16,19,25]$. In einer retrospektiven Studie untersuchten Frankle et al. [6] die Unterschiede im klinischen Ergebnis von Patientinnen über 65 Jahre, die mit einer Osteosynthese $(n=12)$ oder einer primären Prothese $(\mathrm{n}=12)$ versorgt worden waren. Die durchschnittliche Operationsdauer war in der Gruppe der mit einer Prothese versorgten Patientinnen deutlich kürzer als in der mit Osteosynthese behandelten Gruppe (78 vs. $146 \mathrm{~min}$ ). In der Prothesengruppe erzielten alle 12 Patientinnen ein sehr gutes $(n=11)$ oder gutes $(n=1)$ Ergebnis im MEPS, während in der mit Osteosynthe- se behandelten Gruppe nur jeweils $4 \mathrm{~Pa}$ tientinnen ein sehr gutes oder gutes klinisches Ergebnis erreichten, bei 1 Patientin war das Ergebnis moderat, bei 3 weiteren schlecht. Bei Letzteren 3 bestanden, im Gegensatz zu den anderen Patientinnen in dieser Gruppe, eine Dauertherapie mit Kortison bei chronisch obstruktiver Lungenerkrankung $(\mathrm{n}=3)$, eine manifeste Osteoporose $(n=2)$ und/oder eine Alkoholkrankheit $(n=1)$. In diesen 3 Fällen musste sekundär eine Prothese implantiert werden (• Tab. 2). Auch wenn die Ergebnisse dieser Studie auf den ersten Blick überzeugend wirken, muss einschränkend festgestellt werden, dass ein hoher Prozentsatz (67\%) der mit einer Prothese versorgten Patientinnen eine rheumatoide Arthritis als Grunderkrankung aufwies und somit eine Selektion stattgefunden hatte.
McKee et al. [19] schlossen 42 Patienten in eine prospektive, randomisierte, kontrollierte Multicenterstudie ein, in der die klinischen Ergebnisse intraartikulärer mehrfragmentärer Humerusfrakturen nach Osteosynthese bzw. Endoprothese untersucht wurden. Insgesamt konnten 40 Patienten ausgewertet werden (20 Patienten/Gruppe). Intraoperativ wurden 5 Patienten aus der Osteosynthesegruppe in die Prothesengruppe konvertiert, da eine Osteosynthese nicht möglich erschien. Es zeigte sich eine um $32 \mathrm{~min}$ geringere Operationszeit bei den mit einer Prothese versorgten Patienten ( $\mathrm{p}=0,001)$. Der MEPS war in dieser Gruppe zu jedem Untersuchungszeitpunkt $(3,6,12,24 \mathrm{Mo}$ nate) signifikant höher als bei den mittels Osteosynthese versorgten Patienten. Der Bewegungsumfang für Flexion und Extension unterschied sich nicht signifikant 


\begin{tabular}{|lll}
\hline Tab. 3 & Vor- und Nachteile von Osteosynthese/Prothese \\
\hline & Osteosynthese & Prothese \\
\hline Vorteile & Erhalt der Biologie & Stabilität \\
& Gute Langzeitergebnisse & Frühfunktionelle Nachbehandlung \\
& Vollbelastung nach Konsolidierung & Kürzere Operationsdauer \\
& & Weniger Bewegungseinschränkungen \\
\hline Nachteile & Instabilität & Langzeitergebnisse ungewiss \\
& Gelenksteife & Lockerung \\
& Osteosyntheseversagen & Keine Belastbarkeit \\
& & Strikte Disziplin des Patienten erforderlich
\end{tabular}

$(\mathrm{p}=0$,18). Im DASH-Score (DASH: ,disabilities of the arm, shoulder and hand") konnte nur nach 6 Wochen und nach 6 Monaten ein signifikanter Unterschied zugunsten der Patienten nach Prothesenversorgung gefunden werden. Beim DASH handelt es sich um einen Fragebogen, bei dem Patienten ihre Fähigkeiten und Einschränkungen anhand von 30 Items mittels einer Skala von 1-5 beurteilen. Die Rohdaten werden in einen Prozentrang von $0 \%$ (keine Einschränkung) bis 100\% (maximale Einschränkung) umgerechnet [7].

\section{Diskussion}

Wertet man die Literatur und die eigenen Erfahrungen mit den beiden Versorgungsoptionen kritisch, ergeben sich für die beiden Verfahren die in $\mathbf{0}$ Tab. 3 aufgeführten Vor- und Nachteile.

\section{Fazit für die Praxis}

Die Entscheidung, ob bei einer mehrfragmentären distalen Humerusfraktur eine Osteosynthese oder eine endoprothetische Versorgung des Ellenbogens durchgeführt werden, wird anhand der Frakturmorphologie, den Grunderkrankungen des Patienten, der Knochenqualität und den individuellen Erfahrungen des Operateurs getroffen.

Anhand der Literatur kann der endoprothetische Ersatz des Ellenbogengelenks nur bei Patienten über 75 Jahre mit einer dislozierten, intraartikulären, nicht rekonstruierbaren Trümmerfraktur des distalen Humerus als Grad-B-Empfehlung gegeben werden [21, 26]. Ferner besteht die Indikation in dieser Altersgruppe, wenn zusätzlich zu einer intraartikulären, dislozierten distalen Humerusfraktur als Grunderkrankungen eine Osteoporose, eine dauerhafte Therapie mit Kortikoiden, ein Diabetes mellitus oder eine rheumatoide Arthritis bestehen. Das Patientenalter alleine stellt keine Entscheidungsgrundlage dar.

\section{Korrespondenzadresse}

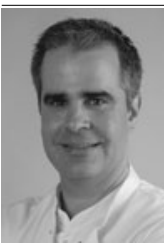

Zentrum für Muskuloskelettale Chirurgie, Klinik und Poliklinik für Unfallchirurgie, Universitätsmedizin, Johannes Gutenberg-Universität Mainz, Langenbeckstraße 1,55101 Mainz sven-oliver.dietz@unimedizinmainz.de

Interessenkonflikt. Der korrespondierende Autor gibt an, dass kein Interessenkonflikt besteht.

\section{Literatur}

1. Ali A, Shahane S, Stanley D (2010) Total elbow arthroplasty for distal humeral fractures: indications, surgical approach, technical tips, and outcome. J Shoulder Elbow Surg 19:53-58

2. Burkhart KJ, Muller LP, Schwarz C et al (2010) Treatment of the complex intraarticular fracture of the distal humerus with the latitude elbow prosthesis. Oper Orthop Traumatol 22:279-298

3. Celli A, Donini M, Minervini C (2008) The use of precontoured plates in the treatment of $\mathrm{C2}-\mathrm{C} 3$ fractures of the distal humerus: clinical experience. Chir Organi Mov 91:57-64

4. Chalidis B, Dimitriou C, Papadopoulos P et al (2009) Total elbow arthroplasty for the treatment of insufficient distal humeral fractures. A retrospective clinical study and review of the literature. Injury 40:582 590

5. Cobb TK, Morrey BF (1997) Total elbow arthroplasty as primary treatment for distal humeral fractures in elderly patients. J Bone Joint Surg Am 79:826-832

6. Frankle MA, Herscovici D Jr, Dipasquale TG et al (2003) A comparison of open reduction and internal fixation and primary total elbow arthroplasty in the treatment of intraarticular distal humerus fractures in women older than age 65. J Orthop Trauma $17: 473-480$

\section{Dr. S.O. Dietz}

7. Germann G, Wind G, Harth A (1999) The DASH (disability of arm-shoulder-hand) questionnaire - a new instrument for evaluating upper extremity treatment outcome. Handchir Mikrochir Plast Chir 31:149-152

8. Greiner S, Haas NP, Bail HJ (2008) Outcome after open reduction and angular stable internal fixation for supra-intercondylar fractures of the distal humerus: preliminary results with the LCP distal humerus system. Arch Orthop Trauma Surg 128:723-729

9. Hessmann MH, Ring DC (2007) Humerus: Humerus, distal. In: Rüedi TP, Buckley RE, Moran CG (Hrsg) AO principles of fracture management. Thieme, Stuttgart New York, S 609-625

10. Holz U (2000) Small bone fragments of great significance. Coronoid process and capitulum humeri. Trauma Berufskrankh 2:S61-S64

11. Huang Jl, Paczas M, Hoyen HA et al (2011) Functional outcome after open reduction internal fixation of intra-articular fractures of the distal humerus in the elderly. J Orthop Trauma 25:259-265

12. Kaiser T, Brunner A, Hohendorff B et al (2011) Treatment of supra- and intra-articular fractures of the distal humerus with the LCP distal humerus plate: a 2-year follow-up. J Shoulder Elbow Surg 20:206-212

13. Kamineni S, Morrey BF (2004) Distal humeral fractures treated with noncustom total elbow replacement. J Bone Joint Surg Am 86-A:940-947

14. Korner J, Lill H, Muller LP et al (2005) Distal humerus fractures in elderly patients: results after open reduction and internal fixation. Osteoporos Int [Suppl 2] 16:S73-S79

15. Kraus E, Harstall R, Borisch N et al (2009) Primary total elbow replacement for complex intra-articular distal humerus fractures. Unfallchirurg 112:692-698

16. Lee KT, Lai CH, Singh S (2006) Results of total elbow arthroplasty in the treatment of distal humerus fractures in elderly Asian patients. J Trauma 61:889-892

17. Liu JJ, Ruan HJ, Wang JG et al (2009) Double-column fixation for type $C$ fractures of the distal humerus in the elderly. J Shoulder Elbow Surg 18:646-651

18. Luegmair M, Timofiev E, Chirpaz-Cerbat JM (2008) Surgical treatment of $A O$ type $C$ distal humeral fractures: internal fixation with a Y-shaped reconstruction (Lambda) plate. J Shoulder Elbow Surg 17:113120

19. Mckee MD, Veillette CJ, Hall JA et al (2009) A multicenter, prospective, randomized, controlled trial of open reduction - internal fixation versus total elbow arthroplasty for displaced intra-articular distal humeral fractures in elderly patients. J Shoulder Elbow Surg 18:3-12

20. Müller M, Allgöwer M, Schnider R (1992) Manual der Osteosynthese. Springer, Berlin Heidelberg New York

21. Nauth A, Mckee MD, Ristevski B et al (2011) Distal humeral fractures in adults. J Bone Joint Surg Am 93:686-700

22. Palvanen M, Kannus P, Niemi S et al (1998) Secular trends in the osteoporotic fractures of the distal humerus in elderly women. Eur J Epidemiol 14:159164

23. Srinivasan K, Agarwal M, Matthews SJ et al (2005) Fractures of the distal humerus in the elderly: is internal fixation the treatment of choice? Clin Orthop Relat Res 222-230

24. Weber O, Burger C, Kabir K et al (2009) Primary endoprosthetic replacement of fractured elbow in elderly patients. Unfallchirurg 112:778-784

25. Weber O, Burger C, Stein G et al (2010) Endoprosthesis for the fractured elbow: uni- and bicompartmental alloarthroplasty of the humero-ulnar joint. Unfallchirurg 113:977-983

26. Wright JG, Einhorn TA, Heckman JD (2005) Grades of recommendation. J Bone Joint Surg Am 87:19091910 